\title{
Editorial
}

Cerebrovascular Diseases
Cerebrovasc Dis 2017;44:I-II

DOI: $10.1159 / 000481448$
Published online: September 22, 2017

\section{Endovascular Therapy: More Questions to be Answered for Best Personalized Interventional Treatment}

Randomized controlled trials have convincingly confirmed the benefit of endovascular mechanical thrombectomy in addition to intravenous thrombolysis in patients with acute large intracranial artery occlusion up to $6 \mathrm{~h}$ after onset of symptoms [1]. Furthermore, the need for intravenous thrombolysis prior to endovascular mechanical thrombectomy has been questioned [2].

More recently, preliminary data have challenged the standard time limits for both procedures up to $24 \mathrm{~h}$, and more studies to demonstrate large study group results are awaited towards an expansion of the therapeutic time windows for all. In addition, refined personalized patient selection criteria are of utmost importance in the interventional procedures to achieve, for example, full recanalization - one of the main parameters associated with early clinical benefit - which depends largely on individual anatomical variability of proximal and intracranial vessels, active collateral capacities, co-existence of atherosclerotic obstructions as well as types of clot formation and obstructions preventing rapid access and retrieving the thrombus [3].

In this current issue of Cerebrovascular Diseases, two articles address these challenges. An excellent paper by Maus et al. [4] from Germany deals with complete large vessel occlusion (CLVO) or incomplete large vessel occlusion (ILVO) on outcome based on a series of 503 consecutive acute stroke patients treated with mechanical thrombectomy: 49 patients (11.3\%) with a median of $\mathrm{Na}$ tional Institutes of Health Stroke Scale (NIHSS) of 11 presented with ILVO and the remainder with CLVO median NIHSS $15(p<0.001)$. Successful reperfusion was reached in 47/49 ILVO versus only 298/381 in CLVO patients ( $p<$ $0.005)$. The favorable clinical outcome at 90 days was $81 \%$ in ILVO versus only $29 \%$ in CLVO $(p<0.001)$; this was independent of age and initial NIHSS. This suggests both better chances only in a minority of patients with ILVO and higher recanalization rates.

Another issue has been raised by Simpkins et al. [5] from Bethesda (NIH), USA, confirming a more recent clinical observation in transient ischemic attack (TIA) patients with fluctuating symptoms after the onset of cerebral ischemia. Chatzikonstantinou et al. [6] had shown that although more sophisticated biomarkers like ABCD2 and ABCD3-I correlated well with early stroke recurrences in TIA patients, easily accessible clinical symptom fluctuations within $24 \mathrm{~h}$ were associated with the worst prognosis to suffer an early stroke after $24 \mathrm{~h}$. This everywhere available clinical biomarker has now been studied in patients with acute cerebral ischemia by Simpkins et al. [5], who analyzed early changes in stroke volume on serial MRIs (baseline, $2 \mathrm{~h}$ post, and $24 \mathrm{~h}$ post onset of symptoms) and showed that neither infarct volume at $24 \mathrm{~h}$ nor per cent of early reperfusion were significantly associated with early neurological outcome; however, early changes 
within $24 \mathrm{~h}$ in stroke volume was the only biomarker that performed significantly different with response to treatment similar to clinical fluctuations.

Both studies illustrate nicely, how badly needed careful individual clinical observations of patients are if treated in an emergency stroke center - technical refinements and large trial-based evidence are certainly worthwhile but should not justify medical ignorance to individual stroke patients' perspectives of risks or benefits associated with interventional treatment, well deriving from careful on-site observations by expert clinicians.

M.G. Hennerici, Mannheim

\section{References}

1 Goyal M, Menon BK, van Zwam WH, Dippel DW, Mitchell PJ, Demchuk AM, et al: Endovascular thrombectomy after large-vessel ischaemic stroke: a meta-analysis of individual patient data from five randomised trials. Lancet 2016;387:1723-1731.

2 Park HK, Chung JW, Hong JH, et al: Preceding intravenous thrombolysis in patients receiving endovascular therapy. Cerebrovasc Dis 2017;44:51-58.
3 Hennerici MG: Predictors of outcome in acute stroke patients: are they reliable and potentially useful? Cerebrovasc Dis 2017;44(12):I-II.

4 Maus V, You S, Kalkan A, Borggrefe J, Kabbasch C, Barnikol UB, Stetefeld H, Dohmen C, Liebig T, Fink GR, Mpotsaris A: Incomplete large vessel occlusions in mechanical thrombectomy: an independent predictor of favorable outcome in ischemic stroke. Cerebrovasc Dis 2017;44:113-121.
5 Simpkins AN, Dias C, Norato G, Kim E, Leigh R; NIH Natural History of Stroke Investigators: Early change in stroke size performs best in predicting response to therapy. Cerebrovasc Dis 2017;44:141-149.

6 Chatzikonstantinou A, Wolf ME, Schaefer A, Hennerici MG: Risk prediction of subsequent early stroke in patients with transient ischemic attacks. Cerebrovasc Dis 2013;36:106109. 